Pacific

Journal of

Mathematics

\title{
EXCEPTIONAL LIE ALGEBRAS, SU(3), AND JORDAN PAIRS
}

\section{PIERO TRUINI}




\title{
EXCEPTIONAL LIE ALGEBRAS, SU(3), AND JORDAN PAIRS
}

\author{
PIERO TRUINI
}

\begin{abstract}
A simple unifying view of the exceptional Lie algebras is presented. The underlying Jordan pair content and role are exhibited. Each algebra contains three Jordan pairs sharing the same Lie algebra of automorphisms and the same external su(3) symmetry. Eventual physical applications and implications of the theory are outlined.
\end{abstract}

\section{Introduction}

The main purpose of this paper is to exhibit a unifying view of all exceptional Lie algebras, which is also very intuitive from the point of view of elementary particle physics. The result is represented by the root diagram in Figure 1.

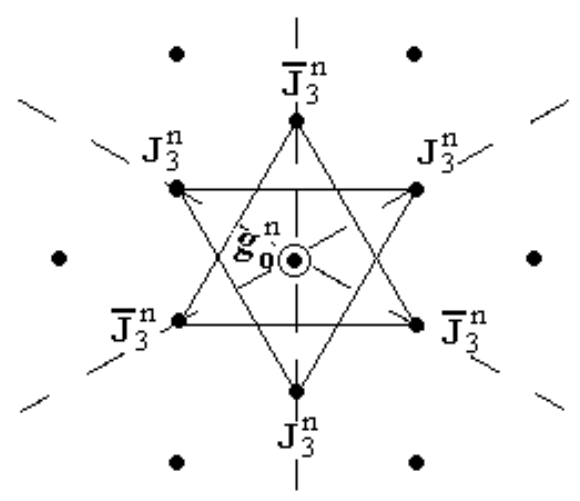

Figure 1. A unifying view of the exceptional Lie algebra roots.

It is a very simple, highly intuitive unifying view of all exceptional Lie algebras and we will use it repeatedly to unfold the largest algebra $\mathbf{e}_{\mathbf{8}}$. The picture shows the projection of the roots of the exceptional Lie algebras on a su(3) plane, recognizable by the dots forming the external hexagon, and it exhibits the Jordan pair content of

Keywords: exceptional Lie algebras, $e_{8}$, Jordan pairs, magic square. 
each exceptional Lie algebra. There are three Jordan pairs $\left(\mathbf{J}_{\mathbf{3}}^{\mathbf{n}}, \overline{\mathbf{J}}_{\mathbf{3}}^{\mathbf{n}}\right)$, each of which lies on an axis symmetrically with respect to the center of the diagram. Each pair doubles a Jordan algebra $\mathbf{J}_{\mathbf{3}}^{\mathbf{n}}$ with involution (the conjugate representation $\overline{\mathbf{J}}_{\mathbf{3}}^{\mathbf{n}}$ ), which is the algebra of $3 \times 3$ Hermitian matrices over $\mathbf{H}$, where $\mathbf{H}=\mathbf{R}, \mathbf{C}, \mathbf{Q}, \mathfrak{C}$ (real, complex, quaternion, and octonion numbers) for $\mathbf{n}=1,2,4,8$. We get $\mathbf{f}_{\mathbf{4}}, \mathbf{e}_{\mathbf{6}}, \mathbf{e}_{\mathbf{7}}$, and $\mathbf{e}_{\mathbf{8}}$ for $\mathbf{n}=1,2,4,8$, respectively ( $\mathbf{g}_{2}$ can be also represented in the same way, with the Jordan algebra reduced to a single element). The three Jordan algebras (and their conjugates) globally behave like a $\mathbf{3}$ (and a $\overline{\mathbf{3}}$ )-dimensional representation of the outer $\mathbf{s u}(\mathbf{3})$. The algebra denoted by $\mathbf{g}_{\mathbf{0}}^{\mathbf{n}}$ in the center (plus the Cartan generator associated with the axis along which the pair lies) is the algebra of the automorphism group of the Jordan pair (the structure group of the corresponding Jordan algebra). In the case of $\mathbf{e}_{\mathbf{8}}$, the algebra $\mathbf{g}_{\mathbf{0}}^{\mathbf{8}}$ is $\mathbf{e}_{\mathbf{6}}$, described by a similar diagram, and we can iterate the process. What we eventually end up with is a decomposition of $\mathbf{e}_{\mathbf{8}}$ entirely given in terms of su(3)'s and Jordan pairs (that we associate to particle-antiparticle pairs): three pairs $\left(\mathbf{J}_{\mathbf{3}}^{\mathbf{8}}, \overline{\mathbf{J}}_{\mathbf{3}}^{\mathbf{8}}\right)$ for three colors of quark-antiquarks, plus three pairs $\left(\mathbf{J}_{\mathbf{3}}^{\mathbf{2}}, \overline{\mathbf{J}}_{\mathbf{3}}^{\mathbf{2}}\right)$, in the colorless $\mathbf{g}_{\mathbf{0}}^{\mathbf{8}}=\mathbf{e}_{\mathbf{6}}$, for three families of leptons-antileptons.

The interest of physicists in the exceptional Lie algebras, and $\mathbf{e}_{\mathbf{8}}$ in particular, is a long-standing tradition, starting from the pioneering work of Gürsey [Frampton et al. 1980] on grand unification, and continuing with [Green and Schwarz 1984; Cremmer 1982; Truini and Biedenharn 1982; Candelas et al. 1985; Gross 1986; Ferrara and Kallosh 1996a; 1996b; Ferrara and Günaydin 1998]. In the effort of unifying all interactions in a consistent quantum theory that includes gravity, the most successful model of string theory is based on $\mathbf{e}_{\mathbf{8}}$; an alternative theory known as loop quantum gravity (see [Rovelli 2004] for an excellent and comprehensive review) has also led towards the exceptional algebras, and $\mathbf{e}_{\mathbf{8}}$ in particular [Lisi et al. 2010].

There is a wide consensus in both mathematics and physics on the appeal of the largest exceptional Lie algebra $\mathbf{e}_{\mathbf{8}}$, considered beautiful by many in spite of its complexity. The best synthesis of this was stated by B. Kostant: "It is easy to arrive at the feeling that a final understanding of the universe must somehow involve $E(8)$, or otherwise put, nature would be foolish not to utilize $E(8)$."

Kostant defines $\mathbf{e}_{\mathbf{8}}$ as "a symphony of 2, 3, 5." In the more modest view of the exceptional algebras I present here the numbers 1, 2, and 3 play the central role: they govern the structure. Number 1 is the whole, the universe of the theory: a Lie algebra. Number 2 stands for pair, and we view it as a particle-antiparticle duality represented by Jordan pairs. Number 3 is the number of colors and the number of families: each Jordan pair occurs three times, in a su(3) symmetry. That is all you need in order to build $\mathbf{e}_{\mathbf{8}}$, as we are going to show.

${ }^{1}$ Quoted by Benjamin Wallace-Wells in "Surfing the Universe", The New Yorker, 21 July 2008. 


\section{Jordan pairs}

In this section we review the concept of a Jordan pair [Loos 1975] (see also [McCrimmon 2004] for an enlightening overview). Jordan algebras have traveled a long journey since their appearance in the 30s [Jordan et al. 1934]. The modern formulation [Jacobson 1966] involves a quadratic map $U_{x} y$ (like $x y x$ for associative algebras) instead of the original symmetric product $x \circ y=x y+y x$. The quadratic map and its linearization $V_{x, y} z=\left(U_{x+z}-U_{x}-U_{z}\right) y$ (like $x y z+z y x$ in the associative case) reveal the mathematical structure of Jordan algebras much more clearly, through the notion of inverse, inner ideal, generic norm, etc. The axioms are:

$$
U_{1}=\text { Id. }, \quad U_{x} V_{y, x}=V_{x, y} U_{x}, \quad U_{U_{x} y}=U_{x} U_{y} U_{x} .
$$

The quadratic formulation led to the concept of Jordan triple systems [Meyberg 1970], an example of which is a pair of modules represented by rectangular matrices. There is no way of multiplying two matrices $x$ and $y$, say $n \times m$ and $m \times n$, respectively, by means of a bilinear product. But one can do it using a product like $x y x$, quadratic in $x$ and linear in $y$. Notice that, like in the case of rectangular matrices, there needs not be a unity in these structures. The axioms are in this case:

$$
U_{x} V_{y, x}=V_{x, y} U_{x}, \quad V_{U_{x} y, y}=V_{x, U_{y} x}, \quad U_{U_{x} y}=U_{x} U_{y} U_{x} .
$$

Finally, a Jordan pair is just a pair of modules $\left(V^{+}, V^{-}\right)$acting on each other (but not on themselves) like a Jordan triple:

$$
\begin{aligned}
U_{x^{\sigma}} V_{y^{-\sigma}, x^{\sigma}} & =V_{x^{\sigma}, y^{-\sigma}} U_{x^{\sigma}}, \\
V_{U_{x} \sigma y^{-\sigma}, y^{-\sigma}} & =V_{x^{\sigma}, U_{y^{-\sigma}} x^{\sigma},}, \\
U_{U_{x} \sigma} y^{-\sigma} & =U_{x^{\sigma}} U_{y^{-\sigma}} U_{x^{\sigma}},
\end{aligned}
$$

where $\sigma= \pm$ and $x^{\sigma} \in V^{+\sigma}, y^{-\sigma} \in V^{-\sigma}$.

Jordan pairs are strongly related to the Tits-Kantor-Koecher construction of Lie algebras [Tits 1962; Kantor 1964; Koecher 1967] (see also the interesting relation to Hopf algebras [Faulkner 2000]):

$$
\mathfrak{L}=J \oplus \operatorname{str}(J) \oplus \bar{J}
$$

where $J$ is a Jordan algebra and $\operatorname{str}(J)=L(J) \oplus \operatorname{Der}(J)$ is the structure algebra of $J$ [McCrimmon 2004]; $L(x)$ is the left multiplication in $J: L(x) y=x \circ y$; and $\operatorname{Der}(J)=[L(J), L(J)]$ is the algebra of derivations of $J$ (the algebra of the automorphism group of $J$ ) [Schafer 1949; 1966].

In the case of (complex) exceptional Lie algebras this construction applies to $\mathbf{e}_{\mathbf{7}}$, with $J=\mathbf{J}_{\mathbf{3}}^{\mathbf{8}}$, the 27-dimensional exceptional Jordan algebra of $3 \times 3$ Hermitian matrices over the octonions, and $\operatorname{str}(J)=\mathbf{e}_{\mathbf{6}} \otimes \mathbf{C}$ (where $\mathbf{C}$ is the complex field). The 
algebra $\mathbf{e}_{\mathbf{6}}$ is called the reduced structure algebra of $J, \operatorname{str}_{0}(J)$, which is namely the structure algebra with the generator corresponding to multiplication by a complex number taken away: $\mathbf{e}_{\mathbf{6}}=L\left(J_{0}\right) \oplus \operatorname{Der}(J)$, with $J_{0}$ denoting the traceless elements of $J$.

The Tits-Kantor-Koecher construction can be generalized as follows: if $\mathfrak{L}$ is a three-graded Lie algebra,

$$
\mathfrak{L}=\mathfrak{L}_{-1} \oplus L_{0} \oplus \mathfrak{L}_{1}, \quad\left[\mathfrak{L}_{i}, \mathfrak{L}_{j}\right] \subset \mathfrak{L}_{i+j},
$$

so that $\left[\mathfrak{L}_{i}, \mathfrak{L}_{j}\right]=0$ whenever $|i+j|>1$, then $\left(\mathfrak{L}_{1}, \mathfrak{L}_{-1}\right)$ forms a Jordan pair, with the Jacobi identity forcing the elements of the pair to act on each other like in a Jordan triple system. The link with the Tits-Kantor-Koecher construction is obtained by letting $J=\mathfrak{L}_{1}$ and $\bar{J}=\mathfrak{L}_{-1}$. The structure group of $J$ is the automorphism group of the Jordan pair $(J, \bar{J})$ and the trilinear product $V_{x^{\sigma}, y^{-\sigma}} z^{\sigma}$ is

$$
V_{x^{\sigma}, y^{-\sigma}} z^{\sigma}=\left[\left[x^{\sigma}, y^{-\sigma}\right], z^{\sigma}\right] .
$$

\section{The Freudenthal-Tits magic square}

The theory of exceptional Lie algebras has had a major advance with the development of two related objects: the Tits construction and the Freudenthal-Tits magic square [Tits 1955; Freudenthal 1959].

The Freudenthal-Tits magic square is a table of Lie algebras related to both Jordan algebras and Hurwitz algebras $\mathbf{H}$, namely the algebras of real $(\mathbf{R})$, complex $(\mathbf{C})$, quaternion $(\mathbf{Q})$, and octonion or Cayley $(\mathfrak{C})$ numbers. In particular the Jordan algebras involved in the magic square are the algebras of $3 \times 3$ Hermitian matrices over $\mathbf{H}$ :

$$
\left(\begin{array}{ccc}
\alpha & a & \bar{b} \\
\bar{a} & \beta & c \\
b & \bar{c} & \gamma
\end{array}\right), \quad \alpha, \beta, \gamma \in \mathbf{C} ; \quad a, b, c \in \mathbf{H} .
$$

We denote them by $\mathbf{J}_{\mathbf{3}}^{\mathbf{n}}$ where $\mathbf{n}=1,2,4,8$ for $\mathbf{H}=\mathbf{R}, \mathbf{C}, \mathbf{Q}, \mathfrak{C}$, respectively. In this paper only complex Lie algebras are considered. Therefore each algebra $\mathbf{H}$ is over the complex field and the $a \rightarrow \bar{a}$ conjugation in (3-1) changes the signs of the imaginary units of $\mathbf{H}$ but does not conjugate the imaginary unit of the underlying complex field. The Freudenthal-Tits magic square is shown in Table 1.

The way the magic square is constructed is due to Tits:

$$
\mathfrak{L}=\operatorname{Der}(\mathbf{H}) \oplus\left(\mathbf{H}_{0} \otimes \mathbf{J}_{\mathbf{0}}\right) \oplus \operatorname{Der}(\mathbf{J}) .
$$

Here the subscript 0 stands for traceless. $\operatorname{Der}(\mathbf{H})$ is the algebra of derivations of $\mathbf{H}$, which is nothing for $\mathbf{H}=\mathbf{R}, \mathbf{C}$, whereas $\operatorname{Der}(\mathbf{Q})=\mathbf{a}_{1}$ and $\operatorname{Der}(\mathfrak{C})=\mathbf{g}_{2}$. 


\begin{tabular}{|lcccc|}
\hline $\mathbf{H} \backslash \mathbf{J}$ & $\mathbf{J}_{3}^{\mathbf{1}}$ & $\mathbf{J}_{\mathbf{3}}^{\mathbf{2}}$ & $\mathbf{J}_{\mathbf{3}}^{\mathbf{4}}$ & $\mathbf{J}_{\mathbf{3}}^{\mathbf{8}}$ \\
$\mathbf{R}$ & $\mathbf{a}_{1}$ & $\mathbf{a}_{2}$ & $\mathbf{c}_{3}$ & $\mathbf{f}_{\mathbf{4}}$ \\
$\mathbf{C}$ & $\mathbf{a}_{2}$ & $\mathbf{a}_{2} \oplus \mathbf{a}_{2}$ & $\mathbf{a}_{5}$ & $\mathbf{e}_{6}$ \\
$\mathbf{Q}$ & $\mathbf{c}_{3}$ & $\mathbf{a}_{5}$ & $\mathbf{d}_{\mathbf{6}}$ & $\mathbf{e}_{7}$ \\
$\mathfrak{C}$ & $\mathbf{f}_{\mathbf{4}}$ & $\mathbf{e}_{\mathbf{6}}$ & $\mathbf{e}_{7}$ & $\mathbf{e}_{\mathbf{8}}$ \\
\hline
\end{tabular}

Table 1. The Freudenthal-Tits magic square.

We also have the following tight link between the entries of the magic square and Jordan structures.

The Lie algebras $\mathbf{g}_{\mathrm{I}}$ in the first row of the magic square are the algebras of derivations of the Jordan algebra in the same column (the corresponding group is the automorphism group of the Jordan algebra):

$$
\begin{gathered}
\mathbf{g}_{\mathrm{I}}=\operatorname{Der}(\mathbf{J}), \quad \text { namely: } \\
\mathbf{a}_{\mathbf{1}}=\operatorname{Der}\left(\mathbf{J}_{\mathbf{3}}^{\mathbf{1}}\right), \quad \mathbf{a}_{\mathbf{2}}=\operatorname{Der}\left(\mathbf{J}_{\mathbf{3}}^{\mathbf{2}}\right), \quad \mathbf{c}_{\mathbf{3}}=\operatorname{Der}\left(\mathbf{J}_{\mathbf{3}}^{\mathbf{4}}\right), \quad \mathbf{f}_{\mathbf{4}}=\operatorname{Der}\left(\mathbf{J}_{\mathbf{3}}^{\mathbf{8}}\right) .
\end{gathered}
$$

The Lie algebras $\mathbf{g}_{\mathrm{II}}$ in the second row are the reduced structure algebras of the Jordan algebra in the same column

$$
\begin{array}{cl}
\mathbf{g}_{\mathrm{II}}=\operatorname{str}_{0}(\mathbf{J}), & \text { namely: } \\
\mathbf{a}_{\mathbf{2}}=\operatorname{str}_{0}\left(\mathbf{J}_{\mathbf{3}}^{\mathbf{1}}\right), \quad \mathbf{a}_{\mathbf{2}} \oplus \mathbf{a}_{\mathbf{2}}=\operatorname{str}_{0}\left(\mathbf{J}_{\mathbf{3}}^{\mathbf{2}}\right), \quad \mathbf{a}_{5}=\operatorname{str}_{0}\left(\mathbf{J}_{\mathbf{3}}^{\mathbf{4}}\right), \quad \mathbf{e}_{\mathbf{6}}=\operatorname{str}_{0}\left(\mathbf{J}_{\mathbf{3}}^{\mathbf{8}}\right) .
\end{array}
$$

The Lie algebras $\mathbf{g}_{\text {III }}$ in the third row are three graded and can be written via the Tits-Kantor-Koecher construction (2-4) or in terms of generalized $2 \times 2$ matrices [Truini et al. 1986]:

$$
\begin{gathered}
\mathbf{g}_{\mathrm{III}}=\mathbf{J} \oplus\left(\mathbf{g}_{\mathrm{II}} \otimes \mathbf{C}\right) \oplus \overline{\mathbf{J}}, \quad \text { namely: } \\
\mathbf{c}_{\mathbf{3}}=\mathbf{J}_{\mathbf{3}}^{\mathbf{1}} \oplus\left(\mathbf{a}_{\mathbf{2}} \oplus \mathbf{C}\right) \oplus \overline{\mathbf{J}}_{\mathbf{3}}^{\mathbf{1}}, \mathbf{a}_{\mathbf{5}}=\mathbf{J}_{\mathbf{3}}^{\mathbf{2}} \oplus\left(\mathbf{a}_{\mathbf{2}} \oplus \mathbf{\mathbf { a } _ { 2 }} \oplus \mathbf{C}\right) \oplus \overline{\mathbf{J}}_{\mathbf{3}}^{\mathbf{2}}, \\
\mathbf{d}_{\mathbf{6}}=\mathbf{J}_{\mathbf{3}}^{\mathbf{4}} \oplus\left(\mathbf{a}_{\mathbf{5}} \oplus \mathbf{C}\right) \oplus \overline{\mathbf{J}}_{\mathbf{3}}^{\mathbf{4}}, \mathbf{e}_{\mathbf{7}}=\mathbf{J}_{\mathbf{3}}^{\mathbf{8}} \oplus\left(\mathbf{e}_{\mathbf{6}} \oplus \mathbf{C}\right) \oplus \overline{\mathbf{J}}_{\mathbf{3}}^{\mathbf{8}} .
\end{gathered}
$$

In our opinion the most natural way of extending a similar analysis to the fourth row is the one described in this paper and represented in Figure 1 or in the expression (4-1) in the next section.

\section{The Jordan pairs inside the exceptional Lie algebras}

In this section we work with the roots of the exceptional Lie algebras and postpone the discussion on explicit representations of the generators to the next section. The notation for the explicit set of roots we use, [Bourbaki 1968] is shown in Table 2 in the Appendix. 


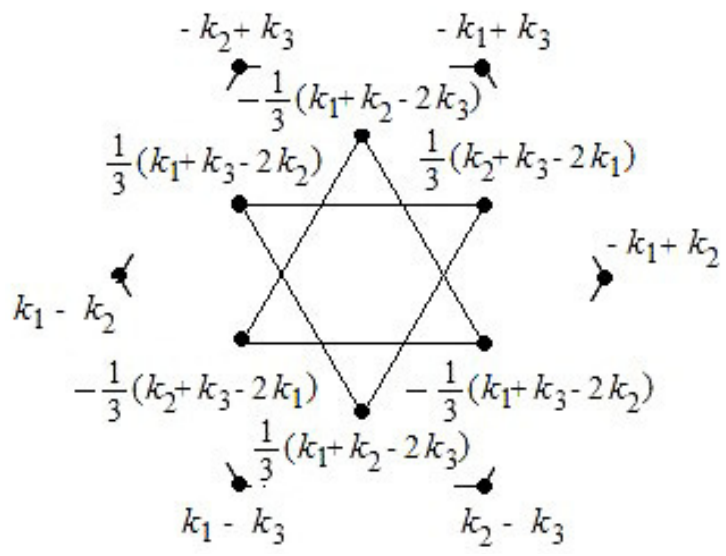

Figure 2. Roots of $\mathbf{g}_{2}$.

The roots can be placed, case by case, as in Figure 1, where they are shown by their projections on the plane of an $\mathbf{a}_{2}$ subalgebra (we use the standard notation $\mathbf{a}_{2}$ for the complexification of $\mathbf{s u}(3)$ ). Notice that $\mathbf{g}_{2}$ itself, as shown in Figure 2, has a root diagram represented by the same dots appearing in Figure 1.

The root diagrams of $\mathbf{f}_{\mathbf{4}}, \mathbf{e}_{\mathbf{6}}, \mathbf{e}_{\mathbf{7}}$, and $\mathbf{e}_{\mathbf{8}}$ are as in Figure 3 . The notation for the Jordan algebras in the figure is the same used in Table 1 for the Freudenthal-Tits magic square: $\mathbf{J}_{\mathbf{3}}^{\mathbf{n}}, \mathbf{n}=1,2,4,8$ is the Jordan algebra of $3 \times 3$ Hermitian matrices over $\mathbf{R}, \mathbf{C}, \mathbf{Q}$, and $\mathfrak{C}$ respectively. The algebra $\mathbf{g}_{\mathbf{0}}^{\mathbf{n}}, \mathbf{n}=1,2,4,8$, is $\mathbf{a}_{2}, \mathbf{a}_{2} \oplus \mathbf{\mathbf { a } _ { 2 }}$, $\mathbf{a}_{5}$, and $\mathbf{e}_{\mathbf{6}}$, respectively; $\mathbf{g}_{\mathbf{0}}^{\mathbf{n}} \oplus \mathbf{C}$ is the algebra of the automorphism group of each Jordan pair $\mathbf{V}^{\mathbf{n}}=\left(\mathbf{J}_{\mathbf{3}}^{\mathbf{n}}, \overline{\mathbf{J}}_{\mathbf{3}}^{\mathbf{n}}\right)$. We associate roots to Jordan pairs and we check that

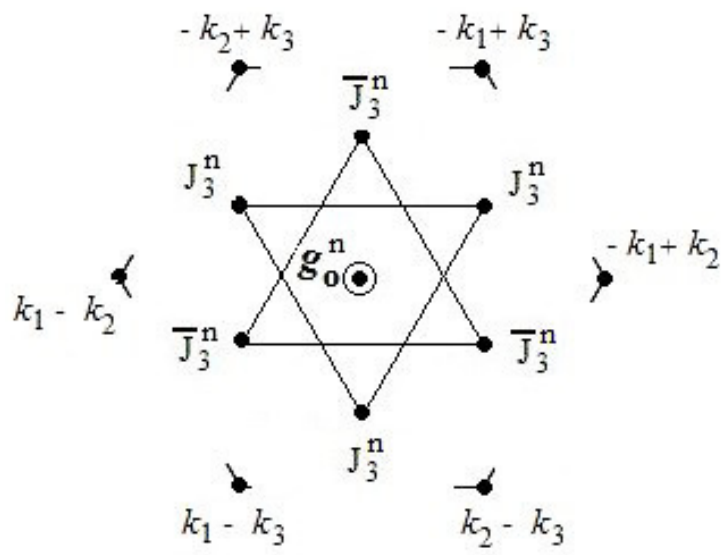

Figure 3. Roots of $\mathbf{f}_{\mathbf{4}}, \mathbf{e}_{\mathbf{6}}, \mathbf{e}_{7}$, and $\mathbf{e}_{\mathbf{8}}$, for $n=1,2,4,8$, respectively, projected on the plane $\Pi$. 


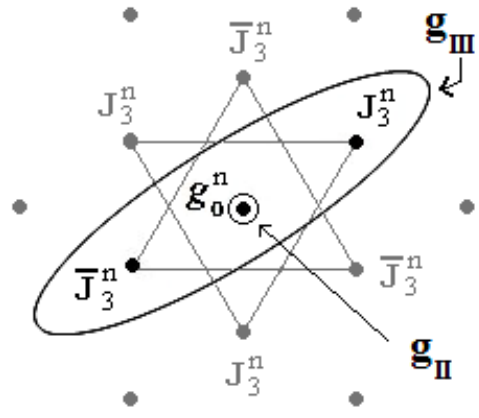

Figure 4. Roots of the exceptional Lie algebras with $\mathbf{g}_{\text {II }}$ and $\mathbf{g}_{\text {III }}$ highlighted.

the projection of these roots lie along an axis, symmetrically with respect to the center. The $\mathbf{C}$ in $\mathbf{g}_{\mathbf{0}}^{\mathbf{n}} \oplus \mathbf{C}$ stands for the complex field and represents the action on $\mathbf{V}^{\mathbf{n}}$ (multiplication by a complex number) of the Cartan generator associated with that axis.

If we write $\mathfrak{L}^{n}$ for $\mathbf{f}_{\mathbf{4}}, \mathbf{e}_{\mathbf{6}}, \mathbf{e}_{7}$, and $\mathbf{e}_{\mathbf{8}}, n=1,2,4,8$, we get the unifying expression

$$
\mathfrak{L}^{n}=\mathbf{a}_{\mathbf{2}} \oplus \mathbf{g}_{\mathbf{0}}^{\mathbf{n}} \oplus 3 \times\left(\mathbf{J}_{\mathbf{3}}^{\mathbf{n}}, \overline{\mathbf{J}}_{\mathbf{3}}^{\mathbf{n}}\right), \quad \text { where } \quad \mathbf{g}_{\mathbf{0}}^{\mathbf{n}}=\operatorname{str}_{0}\left(\mathbf{J}_{\mathbf{3}}^{\mathbf{n}}\right) .
$$

This is not only a unifying view of the exceptional Lie algebras, but also, in our opinion, a natural way of looking at the fourth row of the magic square. Notice that $\mathbf{g}_{\mathbf{0}}^{\mathbf{n}}$ is the Lie algebra in the second row $\left(\mathbf{g}_{\mathrm{II}}\right)$, at the same column of $\mathfrak{L}^{n}$ and that $\mathbf{g}_{\mathbf{0}}^{\mathbf{n}} \oplus \mathbf{C} \oplus \mathbf{V}^{\mathbf{n}}$ is the Lie algebra in the third row ( $\left.\mathbf{g}_{\text {III }}\right)$, same column, for any of the three Jordan pairs $\mathbf{V}^{\mathbf{n}}$ in $\mathfrak{L}^{n}$ (Figure 4).

We explicitly show in the Appendix the roots associated with a Jordan algebra in Figure 3. In particular we will pick the one whose projection on the plane $\Pi$ is $\frac{1}{3}\left(k_{2}+k_{3}-2 k_{1}\right)$ (see Figure 2 for this vector), that is, the highest weight in the 3-dimensional representation of $\mathbf{s u}(3) \sim \mathbf{a}_{2}$. We will refer to this Jordan algebra as the highest-weight (HW) $\mathbf{J}_{\mathbf{3}}^{\mathbf{n}}$. The other Jordan algebras are obtained by a permutation of indexes and their conjugate ones by a change of sign.

Let us explain why we say that certain roots correspond to a Jordan pair. The reason lies in the Tits-Kantor-Koecher construction (2-4), which is related to the third row of the Freudenthal-Tits magic square. There is only one way of realizing the embedding $\mathbf{g}_{\mathrm{II}} \subset \mathbf{g}_{\mathrm{III}} \subset \mathfrak{L}^{n}$ so that the $\left(\mathbf{J}_{\mathbf{3}}^{\mathbf{n}}, \overline{\mathbf{J}}_{\mathbf{3}}^{\mathbf{n}}\right)$ modules for $\mathbf{g}_{\mathrm{II}}$ lie on parallel spaces at the same distance along a fixed axis. This is precisely the way we will describe the Jordan pair content of the algebras and this shows the uniqueness of the construction. We know from the three grading structure of $\mathbf{g}_{\text {III }}$ that the pair $\left(\mathbf{J}_{\mathbf{3}}^{\mathbf{n}}, \overline{\mathbf{J}}_{\mathbf{3}}^{\mathbf{n}}\right)$ is indeed a Jordan pair and that $\operatorname{str}\left(\mathbf{J}_{\mathbf{3}}^{\mathbf{n}}\right)=\mathbf{g}_{\text {II }} \oplus \mathbf{C}$ is the Lie algebra of the automorphism group of the Jordan pair. This proves that the Jordan structures we have referred to so far are indeed so. 
In the Appendix, the four exceptional algebras $\mathbf{f}_{\mathbf{4}}, \mathbf{e}_{\mathbf{6}}, \mathbf{e}_{\mathbf{7}}$, and $\mathbf{e}_{\mathbf{8}}$ are examined case by case. For each we show:

(1) the roots associated with the HW Jordan algebra $\mathbf{J}_{\mathbf{3}}^{\mathbf{n}}$,

(2) the roots associated with $\mathbf{g}_{\mathbf{0}}^{\mathbf{n}}=\mathbf{g}_{\mathrm{II}}$, and

(3) the nested Jordan pairs.

4.1. The geometry of the Jordan pair $\mathrm{V}$ and of $\mathrm{g}_{\mathrm{III}}=\mathrm{g}_{\mathrm{II}} \oplus \mathrm{C} \oplus \mathrm{V}$. The root vectors of the HW $\mathbf{J}_{\mathbf{3}}^{\mathbf{n}}$ all lie on a $(r-2)$-dimensional space $\Sigma^{+}$, where $r$ is the rank of the exceptional Lie algebra. The space $\Sigma^{+}$is parallel to the $(r-2)$-dimensional space $\Sigma^{0}$ on which the $\mathbf{g}_{\mathbf{0}}^{\mathbf{n}}$ roots lie, and to the $(r-2)$-dimensional space $\Sigma^{-}$on which the roots of the $\overline{\mathbf{J}}_{\mathbf{3}}^{\mathbf{n}}$ opposite to the HW $\mathbf{J}_{\mathbf{3}}^{\mathbf{n}}$ lie. Both spaces $\Sigma^{ \pm}$have the same distance $\frac{\sqrt{6}}{3}$ from $\Sigma^{\mathbf{0}}$, but lie on opposite sides with respect to it.

This is shown in Figure 5, in the case of $\mathbf{f}_{\mathbf{4}}$. The two $\mathbf{J}_{\mathbf{3}}^{\mathbf{1}}$ form a Jordan pair of conjugate $\mathbf{a}_{2}$-representations $(6, \overline{6})$. The roots on the three planes form the root diagram of $\mathbf{c}_{3}$.

This Jordan pair is clearly visible in the figure. The Lie algebra of the automorphism group of this pair is $\mathbf{a}_{\mathbf{2}} \oplus \mathbf{C}$ where $\mathbf{C}$ is the complex linear span of the Cartan generator associated with the axis along the vector $\frac{1}{3}\left(k_{2}+k_{3}-2 k_{1}\right)$ which is precisely the direction of the Jordan pair in Figure 3. All the points of the HW $\mathbf{J}_{\mathbf{3}}^{\mathbf{1}}$ (respectively, $\overline{\mathbf{J}}_{\mathbf{3}}^{\mathbf{1}}$, opposite to it with respect to the center of Figure 3) project on the point $\frac{1}{3}\left(k_{2}+k_{3}-2 k_{1}\right)$ (respectively, $\left.-\frac{1}{3}\left(k_{2}+k_{3}-2 k_{1}\right)\right)$ in the plane of Figure 3 .

There is only one way of embedding a $\mathbf{c}_{3}$ subalgebra within $\mathbf{f}_{\mathbf{4}}$ so that the $(6, \overline{6})$ modules for $\mathbf{a}_{2}$ lie on parallel planes at the same distance along a fixed axis. This is precisely the way we have described above and this shows its uniqueness. We know from the three grading structure of $\mathbf{c}_{\mathbf{3}}$ that the pair $\left(\mathbf{J}_{\mathbf{3}}^{\mathbf{1}}, \overline{\mathbf{J}}_{\mathbf{3}}^{\mathbf{1}}\right)$ is indeed a Jordan

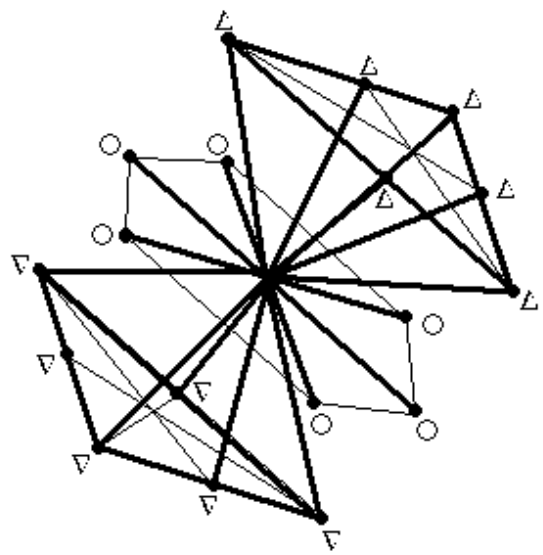

Figure 5. Root digram of $\mathbf{c}_{3}$ showing $\mathbf{a}_{2}$ and the Jordan pair $(6, \overline{6})$. 
pair and that $\operatorname{str}(\mathbf{J})(=\mathbf{a} 2 \oplus \mathbf{C}$ in this case) is the Lie algebra of the automorphism group of the Jordan pair.

By a cyclic permutation of the indexes of $k_{1}, k_{2}$, and $k_{3}$ we obtain an analogous result for the other two Jordan pairs, all sharing the same $\mathbf{a}_{2}$ roots for the algebra $\mathbf{g}_{\mathbf{0}}^{\mathbf{1}}$, but with different orientations of the axis defining $\mathbf{C}$ along the vectors $\frac{1}{3}\left(k_{1}+\right.$ $\left.k_{3}-2 k_{2}\right)$ and $\frac{1}{3}\left(k_{1}+k_{2}-2 k_{3}\right)$. We get in four dimensions three copies of $\mathbf{c}_{3}$ all sharing the same $\mathbf{a}_{2}$. All the spaces spanned by the three Jordan pairs are parallel to the space $\Sigma^{\mathbf{0}}$, and all at the same distance $\pm \frac{\sqrt{6}}{3}$ from it. Notice that in $r$ dimensions there are an infinite number of $(r-2)$-dimensional spaces parallel to a given one, all at the same distance from it.

We get exactly the same feature for the other exceptional Lie algebras, with the Lie algebras of the second and third rows of the magic square playing the same role as for $\mathbf{f}_{\mathbf{4}}$.

\section{Representations}

I briefly sketch in this section a possible representation of the $\mathbf{e}_{\mathbf{8}}$ algebra which exhibits its Jordan pair content.

The way I would represent $\mathbf{e}_{8}$ is a development of the representation of $\mathbf{e}_{7}$ through generalized $2 \times 2$ matrices, shown in [Truini et al. 1986]. The starting point of that paper is the representation of the quaternion algebra through Pauli matrices, which leads directly to the three grading of $\mathbf{e}_{7}$. In the case of $\mathbf{e}_{\mathbf{8}}$ a suitable representation of the octonions is via the Zorn matrices [Zorn 1933; Loos et al. 2008], which exhibit the $(3, \overline{3})$ structure that we can extend to the Jordan pair content of $\mathbf{e}_{\mathbf{8}}$ and to the action on the $(3, \overline{3})$ modules of the external $\mathbf{a}_{2}$ in Figure 3.

The guidelines go as follows:

- Represent the octonions as Zorn matrices.

- Extend the Zorn matrices to represent $\operatorname{Der}(\mathfrak{C})=\mathbf{g}_{\mathbf{2}}$.

- Combine the extended Zorn matrices with the Tits construction (3-2).

- Decompose the representation of $\mathbf{e}_{\mathbf{6}}$ to finally get $\mathbf{e}_{\mathbf{8}}$ in terms of Jordan pairs and $\mathbf{a}_{2}$ 's only.

If $a \in \mathfrak{C}$ we write $a=a_{0}+\sum_{k=1}^{7} a_{k} u_{k}$ where $a_{\ell} \in \mathbf{C}$ for $\ell=0, \ldots, 7$ and $u_{1}, \ldots, u_{7}$ are the octonionic imaginary units.

Let us denote by $i$ the imaginary unit in $\mathbf{C}$. We introduce two idempotent elements

$$
\rho_{ \pm}=\frac{1}{2}\left(1 \pm i u_{7}\right)
$$

and six nilpotent elements

$$
\varepsilon_{k}^{ \pm}=\rho^{ \pm} u_{k}, \quad k=1,2,3 .
$$


The Zorn representation of $a \in \mathfrak{C}$ is:

$$
a=\alpha^{+} \rho^{+}+\alpha^{-} \rho^{-}+\sum_{k}\left(\alpha_{k}^{+} \varepsilon_{k}^{+}+\alpha_{k}^{-} \varepsilon_{k}^{-}\right) \leftrightarrow\left[\begin{array}{cc}
\alpha^{+} & A^{+} \\
A^{-} & \alpha^{-}
\end{array}\right],
$$

where $A^{ \pm} \in \mathbf{C}^{3}$ have vector components $\alpha_{k}^{ \pm}, k=1,2,3$, and the octonionic multiplication is a generalization of matrix multiplication:

$$
\begin{aligned}
a b & \leftrightarrow\left[\begin{array}{ll}
\alpha^{+} & A^{+} \\
A^{-} & \alpha^{-}
\end{array}\right]\left[\begin{array}{ll}
\beta^{+} & B^{+} \\
B^{-} & \beta^{-}
\end{array}\right] \\
& =\left[\begin{array}{cc}
\alpha^{+} \beta^{+}+A^{+} \cdot B^{-} & \alpha^{+} B^{+}+\beta^{-} A^{+}+A^{-} \times B^{-} \\
\alpha^{-} B^{-}+\beta^{+} A^{-}+A^{+} \times B^{+} & \alpha^{-} \beta^{-}+A^{-} \cdot B^{+}
\end{array}\right],
\end{aligned}
$$

with $A^{ \pm} \cdot B^{\mp}=-\alpha_{k}^{ \pm} \beta_{k}^{\mp}$ and where $A, B \rightarrow A \times B$ is the standard vector product in $\mathbf{C}^{3}$.

The next step is to write the Lie algebra $\mathbf{g}_{2}$ using an extension of the Zorn matrices and their multiplication rule with an $\mathbf{a}_{2}$ matrix replacing $\alpha^{+}$. This representation shows $\mathbf{g}_{2}$ as $\mathbf{a}_{2}$ plus its modules $(3, \overline{3})$.

Finally, let me outline how the Tits construction fits into this picture. The idea is to write

$$
\mathbf{e}_{\mathbf{8}}=\operatorname{Der}(\mathfrak{C}) \oplus \mathfrak{C}_{0} \otimes \mathbf{J}_{0}^{8} \oplus \operatorname{Der}\left(\mathbf{J}^{8}\right)=\mathfrak{L}_{0} \oplus \sum_{ \pm k} \mathfrak{L}_{ \pm k}, \quad k=1,2,3,
$$

where

$\mathfrak{L}_{0}=D_{7} \oplus i u_{7} \otimes \mathbf{J}_{0}^{8} \oplus \operatorname{Der}\left(\mathbf{J}^{8}\right) \quad$ and $\quad \mathfrak{L}_{ \pm k}=d_{k}^{ \pm} D_{k}^{ \pm} \oplus \alpha_{k}^{ \pm} \varepsilon_{k}^{ \pm} \otimes \mathbf{J}_{0}^{8}, \quad d_{k}^{ \pm}, \alpha_{k}^{ \pm} \in \mathbf{C}$.

Here $\mathbf{J}^{8} \equiv \mathbf{J}_{\mathbf{3}}^{\mathbf{8}}$ and $\mathbf{J}_{0}^{8}$ is a traceless $\mathbf{J}_{\mathbf{3}}^{\mathbf{8}}$ matrix; $D_{7}=\mathbf{a}_{\mathbf{2}}$ is the subalgebra of derivations leaving the imaginary unit $u_{7}$ fixed; and $D_{k}^{ \pm}= \pm \frac{3}{2} D_{i u_{7}, \varepsilon_{k}^{ \pm}}$is a derivation:

$$
D_{a, b} c=\frac{1}{3}[[a, b], c]-(a, b, c), \quad(a, b, c)=(a b) c-a(b c) .
$$

We identify $a \otimes x$ with $a_{z} \otimes x$, where $a_{z}$ is the Zorn matrix representation of $a$ and $\operatorname{Der}_{k}^{ \pm}$with the corresponding Zorn matrix representation of $\varepsilon_{k}^{ \pm}$. We use the complex parameters $d_{k}^{ \pm}$in order to provide the trace to $\mathbf{J}_{0}$.

The chain of implications, starting from the Tits construction, would be like this:

$$
\begin{aligned}
\mathbf{e}_{8} & =\operatorname{Der}(\mathfrak{C}) \oplus \mathfrak{C}_{0} \otimes \mathbf{J}_{0}^{8} \oplus \operatorname{Der}\left(\mathbf{J}^{8}\right) \\
& =\mathbf{a}_{2}^{\mathbf{c}} \oplus \alpha_{k}^{ \pm} \varepsilon_{k}^{ \pm} \otimes \mathbf{J}_{0}^{8} \oplus d_{k}^{ \pm} \operatorname{Der}_{k}^{ \pm} \oplus\left(i u_{7}\right) \otimes \mathbf{J}_{0}^{8} \oplus \operatorname{Der}\left(\mathbf{J}^{8}\right) \\
& =\mathbf{a}_{2}^{\mathbf{c}} \oplus \alpha_{k}^{ \pm} \varepsilon_{k}^{ \pm} \otimes \mathbf{J}^{8} \oplus \operatorname{Der}\left(\mathfrak{C}^{\mathfrak{c}}\right) \oplus \mathfrak{C}_{0} \otimes \mathbf{J}_{0}^{2} \oplus \operatorname{Der}\left(\mathbf{J}^{2}\right) \\
& =\mathbf{a}_{2}^{\mathbf{c}} \oplus \alpha_{k}^{ \pm} \varepsilon_{k}^{ \pm} \otimes \mathbf{J}^{8} \oplus \mathbf{a}_{2}^{\mathbf{f}} \oplus \alpha_{k}^{ \pm} \varepsilon_{k}^{ \pm} \otimes \mathbf{J}^{2} \oplus\left(i u_{7}\right) \otimes \mathbf{J}_{0}^{2} \oplus \operatorname{Der}\left(\mathbf{J}^{2}\right) \\
& =\mathbf{a}_{2}^{\mathbf{c}} \oplus \mathbf{a}_{2}^{\mathbf{f}} \oplus \mathbf{a}_{\mathbf{2}}^{\mathbf{g}_{1}} \oplus \mathbf{a}_{\mathbf{2}}^{\mathbf{g}_{2}} \oplus 3 \times\left(\mathbf{J}^{8}, \overline{\mathbf{J}}^{8}\right) \oplus 3 \times\left(\mathbf{J}^{2}, \overline{\mathbf{J}}^{2}\right) .
\end{aligned}
$$

Work is still in progress along these lines and will appear in a forthcoming paper. 


\section{Elementary particle physics}

If we look at the decomposition (5-4) (see also (A.7) in the Appendix) we are led to interpret the labels $\mathbf{c}$ as color and $\mathbf{f}$ as flavor. In this interpretation the three pairs $\left(\mathbf{J}_{\mathbf{3}}^{\mathbf{8}}, \overline{\mathbf{J}}_{\mathbf{3}}^{\mathbf{8}}\right)$ accommodate the quarks in three colors of particles-antiparticles, whereas the three pairs $\left(\mathbf{J}_{\mathbf{3}}^{\mathbf{2}}, \overline{\mathbf{J}}_{\mathbf{3}}^{\mathbf{2}}\right)$ sitting in the colorless $\mathbf{g}_{\mathbf{0}}^{\mathbf{8}}$ accommodate the three families of leptons-antileptons. Including spin, each particle must appear with four different degrees of freedom: left (up and down) and right (up and down), except, possibly, for the neutrino, which could be a Majorana neutrino and be only left-handed. We can therefore put six (quarks, antiquarks) in a (say) blue $\left(\mathbf{J}_{\mathbf{3}}^{\mathbf{8}}, \overline{\mathbf{J}}_{\mathbf{3}}^{\mathbf{8}}\right)$. We can make them coincide with three octonions: one for blue up-down quarks, one for blue charm-strange quarks, one for blue top-bottom quarks. We are left with three extra degrees of freedom. In the same fashion, we can put a family of leptons-antileptons pairs in $\left(\mathbf{J}_{\mathbf{3}}^{\mathbf{2}}, \overline{\mathbf{J}}_{\mathbf{3}}^{\mathbf{2}}\right)$ by letting the six off-diagonal degrees of freedom of be the electron and a Majorana neutrino, and analogously for the families of the muon and $\tau$ leptons. Again we are left with three extra degrees of freedom, which reduce to only one in the case where right-handed neutrinos are included.

Let us review the explicit form of the roots (see the Appendix) according to this interpretation.

Quarks of color $\mathbf{c}=1,2,3$ (corresponding antiquarks have reversed signs):

$$
\begin{gathered}
-k_{c} \pm k_{j}, \quad j=4, \ldots, 8, \quad-k_{c}+k_{1}+k_{2}+k_{3}, \\
-k_{c}+\frac{1}{2}\left(k_{1}+k_{2}+k_{3} \pm k_{4} \pm k_{5} \pm k_{6} \pm k_{7} \pm k_{8}\right) \quad \text { (even \# of }+ \text { signs). }
\end{gathered}
$$

Leptons in the family $\mathbf{f}=4,5,6$ (corresponding antileptons have reversed signs):

$$
\begin{gathered}
-k_{f} \pm k_{j}, \quad j=7,8, \quad-k_{f}+k_{4}+k_{5}+k_{6}, \\
-k_{f}+\frac{1}{2}\left[ \pm\left(k_{1}+k_{2}+k_{3}\right)+k_{4}+k_{5}+k_{6} \pm k_{7} \pm k_{8}\right] \quad \text { (even \# of }+ \text { signs). }
\end{gathered}
$$

$\mathbf{a}_{2}^{\mathbf{c}}: \pm\left(k_{i}-k_{j}\right), i<j=1,2,3$.

$\mathbf{a}_{2}^{\mathbf{f}}: \pm\left(k_{i}-k_{j}\right), i<j=4,5,6$.

$\mathbf{a}_{2}^{\mathbf{g}_{1}}: \pm\left(k_{7}+k_{8}\right), \pm \frac{1}{2}\left(k_{1}+k_{2}+k_{3}+k_{4}+k_{5}+k_{6}-k_{7}-k_{8}\right) \pm \frac{1}{2}\left(k_{1}+k_{2}+k_{3}+k_{4}+\right.$ $\left.k_{5}+k_{6}+k_{7}+k_{8}\right)$.

$\mathbf{a}_{2}^{\mathbf{g}_{2}}: \pm\left(k_{7}-k_{8}\right), \pm \frac{1}{2}\left(-k_{1}-k_{2}-k_{3}+k_{4}+k_{5}+k_{6}-k_{7}+k_{8}\right) \pm \frac{1}{2}\left(-k_{1}-k_{2}-k_{3}+\right.$ $\left.k_{4}+k_{5}+k_{6}+k_{7}-k_{8}\right)$.

What physics should a theory with an $\mathbf{e}_{\mathbf{8}}$ symmetry describe? Certainly a very high-energy physics, far beyond our present experience and our experimental reach. It could relate to a string theory, like the heterotic one, since we are dealing with a complex Lie algebra hence an $\mathbf{e}_{8} \times \mathbf{e}_{8}$ algebra over $\mathbf{R}$. It could extend to supersymmetry, although the $\mathbf{e}_{\mathbf{8}}$ symmetry is so beautiful as it stands that one 


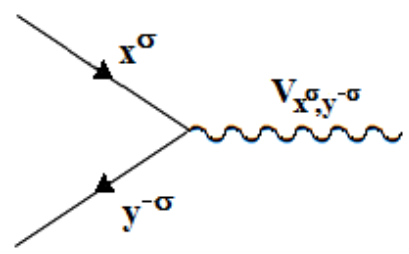

Figure 6. An elementary interaction, viewed as a Feynman diagram.

should force such an extension into the theory: $\mathbf{e}_{8}$, in the view I am presenting here, shows particle-antiparticle pairs, the Jordan pairs, in the right number of colors and families, plus their symmetries, which in turn are generated by the pairs themselves, through the trilinear map $z^{\sigma} \rightarrow V_{x^{\sigma}, y^{-\sigma}} z^{\sigma}$. Besides, another peculiarity contributes to the beauty of $\mathbf{e}_{\mathbf{8}}$ : its lowest-dimensional irreducible representation is the adjoint representation.

My personal point of view is that, at such a high energy, at or beyond the Planck scale, the picture of spacetime has to be radically changed. I can hardly make any sense of the fact that such an energetic particle is sitting on a background spacetime, if I think that general relativity taught us that spacetime is in fact dynamical. I would rather view that particle as feeling only (quantum) interactions, including one that leads to gravity, to be accommodated within $\mathbf{a}_{2}^{\mathbf{g}_{1}} \oplus \mathbf{a}_{2}^{\mathbf{g}_{2}}$. I would still view an elementary interaction being described by an elementary Feynman diagram involving the trilinear map, as depicted in Figure 6, but with no question of point or extended particle, simply because the underlying spacetime geometry is not there: there is only a, let us say, background independent spectral theory.

In this view the classical spacetime is a byproduct of the interactions, obtained by taking very rough approximations. It is as far from the interactions exchanged by elementary particles at the Planck scale, as the Planck scale is far from our experience.

The aim of developing along these lines a physical theory that could not possibly rely on any direct confirmation, is to find a consistent quantum theory of gravity together with the other known basic interactions. As Carlo Rovelli says [2004]: "the difficulty is not to discriminate among many complete and consistent quantum theories of gravity. We would be content with one."

This is, of course, far beyond the scope of the present paper, since no physics has been spoken here besides these mere speculations.

\section{Appendix}

The explicit set of roots we use is shown in Table 2 [Bourbaki 1968]; $\left\{k_{i}, i=\right.$ $1, \ldots, 8\}$ denotes an orthonormal basis in $\mathbf{R}^{8}$. 


\begin{tabular}{|c|c|c|}
\hline $\mathfrak{L}$ & Roots $\left\{k_{i}, i=1, \ldots 8\right\}$ an orthonormal basis in $\mathbf{R}^{8}$ & \# of roots \\
\hline $\mathbf{g}_{2}$ & $\begin{array}{l}\left(k_{i}-k_{j}\right), \quad i \neq j=1,2,3 \\
\pm \frac{1}{3}\left(-2 k_{i}+k_{j}+k_{l}\right), \quad i \neq j \neq l=1,2,3\end{array}$ & $\begin{array}{c}12 \\
6 \\
6\end{array}$ \\
\hline $\mathbf{f}_{4}$ & $\begin{array}{l} \pm k_{i}, \quad i=1, \ldots, 4 \\
\pm k_{i} \pm k_{j}, \quad i \neq j=1, \ldots, 4 \\
\frac{1}{2}\left( \pm k_{1} \pm k_{2} \pm k_{3} \pm k_{4}\right)\end{array}$ & $\begin{array}{c}48 \\
8 \\
4 \times\left(\begin{array}{l}4 \\
2\end{array}\right)=24 \\
2^{4}=16\end{array}$ \\
\hline $\mathbf{e}_{6}$ & $\begin{array}{l} \pm k_{i} \pm k_{j}, \quad i \neq j=1, \ldots, 5 \\
\frac{1}{2}\left( \pm k_{1} \pm k_{2} \pm k_{3} \pm k_{4} \pm k_{5} \pm \sqrt{3} k_{6}\right)^{*} \\
* \text { odd number of }+ \text { signs }\end{array}$ & $\begin{array}{c}72 \\
4 \times\left(\begin{array}{l}5 \\
2\end{array}\right)=40 \\
2^{5}=32\end{array}$ \\
\hline $\mathbf{e}_{7}$ & $\begin{array}{l} \pm \sqrt{2} k_{7}, \\
\pm k_{i} \pm k_{j}, \quad i \neq j=1, \ldots, 6 \\
\frac{1}{2}\left( \pm k_{1} \pm k_{2} \pm k_{3} \pm k_{4} \pm k_{5} \pm k_{6} \pm \sqrt{2} k_{7}\right)^{*} \\
* \text { even number of }+\frac{1}{2}\end{array}$ & $\begin{array}{c}126 \\
2 \\
4 \times\left(\begin{array}{l}6 \\
2\end{array}\right)=60 \\
2^{6}=64\end{array}$ \\
\hline $\mathbf{e}_{8}$ & $\begin{array}{l} \pm k_{i} \pm k_{j}, \quad i \neq j=1, \ldots, 8 \\
\frac{1}{2}\left( \pm k_{1} \pm k_{2} \pm k_{3} \pm k_{4} \pm k_{5} \pm k_{6} \pm k_{7} \pm k_{8}\right)^{*} \\
* \text { even number of }+ \text { signs }\end{array}$ & $\begin{array}{c}\mathbf{2 4 0} \\
4 \times\left(\begin{array}{l}8 \\
2\end{array}\right)=112 \\
2^{7}=128\end{array}$ \\
\hline
\end{tabular}

Table 2. The roots of the exceptional Lie algebras.

\section{A.1 $\mathbf{f}_{4}$.}

A.1.1 The roots associated with the $H W \mathbf{J}_{\mathbf{3}}^{\mathbf{1}}$.

(A.1) $\quad-k_{1}, \quad-k_{1} \pm k_{4}, \quad \frac{1}{2}\left(-k_{1}+k_{2}+k_{3} \pm k_{4}\right), \quad k_{2}+k_{3}$.

A.1.2 The roots associated with $\mathbf{g}_{\mathrm{II}}=\mathbf{g}_{\mathbf{0}}^{\mathbf{1}}$.

$$
\pm k_{4}, \quad \pm \frac{1}{2}\left(k_{1}+k_{2}+k_{3} \pm k_{4}\right) .
$$

A.1.3 Nested Jordan pairs. If we dig inside $\mathbf{g}_{\mathbf{0}}^{\mathbf{1}}$ we find another Jordan pair plus the Lie algebra of its automorphism group: these are a $(2, \overline{2})$ of $\mathbf{a}_{1}$ plus $\mathbf{a}_{1} \oplus \mathbf{C}$ making up, all together, $\mathbf{a}_{2}$.

\section{A.2 $\mathrm{e}_{6}$.}


A.2.1 The roots associated with the $H W \mathbf{J}_{\mathbf{3}}^{\mathbf{2}}$.

$$
\begin{gathered}
-k_{1} \pm k_{4}, \quad-k_{1} \pm k_{5}, \quad k_{2}+k_{3}, \\
\frac{1}{2}\left(-k_{1}+k_{2}+k_{3}+k_{4}-k_{5}-\sqrt{3} k_{6}\right), \\
\frac{1}{2}\left(-k_{1}+k_{2}+k_{3}+k_{4}+k_{5}+\sqrt{3} k_{6}\right), \\
\frac{1}{2}\left(-k_{1}+k_{2}+k_{3}-k_{4}+k_{5}-\sqrt{3} k_{6}\right), \\
\frac{1}{2}\left(-k_{1}+k_{2}+k_{3}-k_{4}-k_{5}+\sqrt{3} k_{6}\right) .
\end{gathered}
$$

A.2.2 The roots associated with $\mathbf{g}_{\mathrm{II}}=\mathbf{g}_{\mathbf{0}}^{\mathbf{2}}$.

$$
\begin{array}{ll}
\mathbf{a}_{\mathbf{2}}^{(\mathbf{1})}: & \\
& \pm\left(k_{4}+k_{5}\right), \\
& \pm \frac{1}{2}\left(k_{1}+k_{2}+k_{3}-k_{4}-k_{5}-\sqrt{3} k_{6}\right), \\
& \pm \frac{1}{2}\left(k_{1}+k_{2}+k_{3}+k_{4}+k_{5}-\sqrt{3} k_{6}\right) . \\
\mathbf{a}_{\mathbf{2}}^{(\mathbf{2})}: \quad & \\
& \pm\left(k_{4}-k_{5}\right), \\
& \pm \frac{1}{2}\left(k_{1}+k_{2}+k_{3}-k_{4}+k_{5}+\sqrt{3} k_{6}\right), \\
& \pm \frac{1}{2}\left(k_{1}+k_{2}+k_{3}+k_{4}-k_{5}+\sqrt{3} k_{6}\right) .
\end{array}
$$

A.2.3 Nested Jordan pairs. If we dig inside $\mathbf{g}_{\mathbf{0}}^{\mathbf{2}}$ we find another Jordan pair plus the Lie algebra of its automorphism group: these are two replicas of a $(2, \overline{2})$ of $\mathbf{a}_{1}$ plus $\mathbf{a}_{1} \oplus \mathbf{C}$ making up, all together, $\mathbf{a}_{2} \oplus \mathbf{\mathbf { a } _ { 2 }}$.

\section{A.3 $\mathrm{e}_{7}$.}

A.3.1 The roots associated with the $H W \mathbf{J}_{\mathbf{3}}^{\mathbf{4}}$.

$$
\begin{aligned}
-k_{1} \pm & k_{4}, \quad-k_{1} \pm k_{5}, \quad-k_{1} \pm k_{6}, \quad k_{2}+k_{3}, \\
& \frac{1}{2}\left(-k_{1}+k_{2}+k_{3}-k_{4}-k_{5}-k_{6} \pm \sqrt{2} k_{7}\right), \\
& \frac{1}{2}\left(-k_{1}+k_{2}+k_{3}-k_{4}+k_{5}+k_{6} \pm \sqrt{2} k_{7}\right), \\
& \frac{1}{2}\left(-k_{1}+k_{2}+k_{3}+k_{4}-k_{5}+k_{6} \pm \sqrt{2} k_{7}\right), \\
& \frac{1}{2}\left(-k_{1}+k_{2}+k_{3}+k_{4}+k_{5}-k_{6} \pm \sqrt{2} k_{7}\right) .
\end{aligned}
$$

A.3.2 The roots associated with $\mathbf{g}_{\mathrm{II}}=\mathbf{g}_{\mathbf{0}}^{\mathbf{4}}$.

$$
\begin{gathered}
\pm k_{4} \pm k_{5}, \quad \pm k_{4} \pm k_{6}, \quad \pm k_{5} \pm k_{6}, \quad \pm \sqrt{2} k_{7}, \\
\left. \pm \frac{1}{2}\left(k_{1}+k_{2}+k_{3} \pm k_{4} \pm k_{5} \pm k_{6} \pm \sqrt{2} k_{7}\right) \quad \text { (even number of }+\frac{1}{2}\right) .
\end{gathered}
$$


A.3.3 Nested Jordan pairs. If we dig inside $\mathbf{g}_{\mathbf{0}}^{\mathbf{4}}=\mathbf{\mathbf { a } _ { 5 }}$ we find the Jordan pair $\left(\mathbf{J}_{\mathbf{3}}^{\mathbf{2}}, \overline{\mathbf{J}}_{\mathbf{3}}^{\mathbf{2}}\right)$ $=(3 \times 3, \overline{3} \times \overline{3})$ plus the Lie algebra of its automorphism group $\mathbf{a}_{2} \oplus \mathbf{a}_{2} \oplus \mathbf{C}$ described in the previous case of $\mathbf{e}_{\mathbf{6}}$.

\section{A.4 e 8 .}

A.4.1 The roots associated with the $H W \mathbf{J}_{3}^{\mathbf{8}}$.

$$
\begin{gathered}
-k_{1} \pm k_{j}, \quad j=4, \ldots, 8, \\
k_{2}+k_{3}, \\
\frac{1}{2}\left(-k_{1}+k_{2}+k_{3} \pm k_{4} \pm k_{5} \pm k_{6} \pm k_{7} \pm k_{8}\right)
\end{gathered}
$$

A.4.2 The roots associated with $\mathbf{g}_{\mathrm{II}}=\mathbf{g}_{\mathbf{0}}^{\mathbf{8}}$. The 72 roots of $\mathbf{g}_{\mathbf{0}}^{\mathbf{8}}=\mathbf{e}_{\mathbf{6}}$ are

$$
\pm k_{i} \pm k_{j}, \quad i, j=4, \ldots, 8,
$$

$$
\pm \frac{1}{2}\left(k_{1}+k_{2}+k_{3} \pm k_{4} \pm k_{5} \pm k_{6} \pm k_{7} \pm k_{8}\right) \quad \text { (even number of }+ \text { signs). }
$$

A.4.3 Nested Jordan pairs. If we dig inside $\mathbf{g}_{\mathbf{0}}^{\mathbf{8}}=\mathbf{e}_{\mathbf{6}}$ we find three Jordan pairs, each of the type $\left(\mathbf{J}_{\mathbf{3}}^{\mathbf{2}}, \overline{\mathbf{J}}_{\mathbf{3}}^{\mathbf{2}}\right)=(3 \times 3, \overline{3} \times \overline{3})$, plus the Lie algebra of the automorphism group of each of them $\mathbf{a}_{\mathbf{2}} \oplus \mathbf{a}_{\mathbf{2}} \oplus \mathbf{C}$ described in the previous case of $\mathbf{e}_{\mathbf{6}}$.

We thus identify four different a a's within es plus six Jordan pairs. Giving different superscripts to the four $\mathbf{a}_{2}$ 's we have:

$$
\begin{aligned}
\mathbf{e}_{\mathbf{8}} & =\mathbf{a}_{\mathbf{2}}^{\mathbf{c}} \oplus 3 \times\left(\mathbf{J}_{\mathbf{3}}^{\mathbf{8}}, \overline{\mathbf{J}}_{\mathbf{3}}^{\mathbf{8}}\right) \oplus \mathbf{g}_{\mathbf{0}}^{\mathbf{8}} \\
& =\mathbf{a}_{\mathbf{2}}^{\mathbf{c}} \oplus 3 \times\left(\mathbf{J}_{\mathbf{3}}^{\mathbf{8}}, \overline{\mathbf{J}}_{\mathbf{3}}^{\mathbf{8}}\right) \oplus \mathbf{a}_{\mathbf{2}}^{\mathbf{f}} \oplus 3 \times\left(\mathbf{J}_{\mathbf{3}}^{\mathbf{2}}, \overline{\mathbf{J}}_{\mathbf{3}}^{\mathbf{2}}\right) \oplus \mathbf{g}_{\mathbf{0}}^{\mathbf{2}} \\
& =\mathbf{a}_{\mathbf{2}}^{\mathbf{c}} \oplus 3 \times\left(\mathbf{J}_{\mathbf{3}}^{\mathbf{8}}, \overline{\mathbf{J}}_{\mathbf{3}}^{\mathbf{8}}\right) \oplus \mathbf{a}_{\mathbf{2}}^{\mathbf{f}} \oplus 3 \times\left(\mathbf{J}_{\mathbf{3}}^{\mathbf{2}}, \overline{\mathbf{J}}_{\mathbf{3}}^{\mathbf{2}}\right) \oplus \mathbf{a}_{\mathbf{2}}^{\mathbf{g}_{1}} \oplus \mathbf{a}_{\mathbf{2}}^{\mathbf{g}_{\mathbf{2}}} .
\end{aligned}
$$

\section{Acknowledgments}

I am very grateful to Professor V. S. Varadarajan for scientific advice and financial support. The discussions we have had together have actually given life to the idea of writing this paper. I also thank Professor Varadarajan and the UCLA Department of Mathematics for their kind hospitality. The visit to the University of California at Los Angeles was made possible by the Istituto Nazionale di Fisica Nucleare, on grant In. Spec. GE 41.

\section{References}

[Bourbaki 1968] N. Bourbaki, Groupes et algèbres de Lie, Chapitres IV-VI, Actualités Scientifiques et Industrielles 1337, Hermann, Paris, 1968. MR 39 \#1590 Zbl 0186.33001

[Candelas et al. 1985] P. Candelas, G. T. Horowitz, A. Strominger, and E. Witten, "Vacuum configurations for superstrings", Nuclear Phys. B 258:1 (1985), 46-74. MR 87k:83091a

[Cremmer 1982] E. Cremmer, "Dimensional reduction in field theory and hidden symmetries in extended supergravity”, pp. xi+489 in Supergravity: Proceedings of the First School (Trieste, 1981), edited by S. Ferrara and J. G. Taylor, Cambridge University Press, Cambridge, 1982. 
[Faulkner 2000] J. R. Faulkner, "Jordan pairs and Hopf algebras", J. Algebra 232:1 (2000), 152-196. MR 2001e:17042 Zbl 1018.17022

[Ferrara and Günaydin 1998] S. Ferrara and M. Günaydin, "Orbits of exceptional groups, duality and BPS states in string theory", Internat. J. Modern Phys. A 13:13 (1998), 2075-2088. MR 99g:81160

[Ferrara and Kallosh 1996a] S. Ferrara and R. Kallosh, "Supersymmetry and attractors", Phys. Rev. D (3) 54:2 (1996), 1514-1524. MR 97e:83101 Zbl 1171.83329

[Ferrara and Kallosh 1996b] S. Ferrara and R. Kallosh, "University of supersymmetry and attractors", Phys. Rev. D 54:2 (1996), 1525-1534.

[Frampton et al. 1980] P. H. Frampton, S. L. Glashow, and A. Yildiz (editors), First Workshop on Grand Unification (Durham, NH, 1980), Lie Groups: History, Frontiers and Applications 11, Math Sci Press, Brookline, MA, 1980. MR 81j:81066

[Freudenthal 1959] H. Freudenthal, "Beziehungen der $\mathfrak{E}_{7}$ und $\mathfrak{E}_{8}$ zur Oktavenebene, VIII-IX", Nederl. Akad. Wetensch. Proc. Ser. A 62 (1959), 447-465. Zbl 0128.15302

[Green and Schwarz 1984] M. B. Green and J. H. Schwarz, "Anomaly cancellations in supersymmetric $D=10$ gauge theory and superstring theory", Phys. Lett. B 149:1-3 (1984), 117-122. MR 85k:81173

[Gross 1986] D. J. Gross, “The heterotic string”, pp. 357-399 in Workshop on unified string theories (Santa Barbara, CA, 1985), edited by M. B. Green and D. Gross, World Scientific, Singapore, 1986. MR 849110

[Jacobson 1966] N. Jacobson, "Structure theory for a class of Jordan algebras", Proc. Nat. Acad. Sci. USA 55 (1966), 243-251. MR 33 \#1337 Zbl 0133.28903

[Jordan et al. 1934] P. Jordan, J. von Neumann, and E. Wigner, "On an algebraic generalization of the quantum mechanical formalism", Ann. of Math. (2) 35:1 (1934), 29-64. MR 1503141 Zbl 0008.42103

[Kantor 1964] I. L. Kantor, "Classification of irreducible transitive differential groups", Dokl. Akad. Nauk SSSR 158 (1964), 1271-1274. In Russian; translated in Sov. Math. Dokl. 5 (1965), 1404-1407. MR 31 \#217 Zbl 0286.17011

[Koecher 1967] M. Koecher, "Imbedding of Jordan algebras into Lie algebras, I", Amer. J. Math. 89 (1967), 787-816. MR 35 \#5480 Zbl 0209.06801

[Lisi et al. 2010] A. G. Lisi, L. Smolin, and S. Speziale, "Unification of gravity, gauge fields and Higgs bosons", J. Phys. A 43:44 (2010), 445401, 10. MR 2011k:81283 Zbl 1202.81226

[Loos 1975] O. Loos, Jordan pairs, Lecture Notes in Mathematics 460, Springer, Berlin, 1975. MR 56 \#3071 Zbl 0301.17003

[Loos et al. 2008] O. Loos, H. P. Petersson, and M. L. Racine, "Inner derivations of alternative algebras over commutative rings", Algebra Number Theory 2:8 (2008), 927-968. MR 2009i:17048 Zbl 1191.17011

[McCrimmon 2004] K. McCrimmon, A taste of Jordan algebras, Springer, New York, 2004. MR 2004i: 17001 Zbl 1044.17001

[Meyberg 1970] K. Meyberg, "Jordan-Tripelsysteme und die Koecher-Konstruktion von Lie-Algebren”, Math. Z. 115 (1970), 58-78. MR 41 \#8482 Zbl 0186.34501

[Rovelli 2004] C. Rovelli, Quantum gravity, Cambridge University Press, Cambridge, 2004. MR 2005m:83049 Zbl 1091.83001

[Schafer 1949] R. D. Schafer, "Inner derivations of non-associative algebras", Bull. Amer. Math. Soc. 55 (1949), 769-776. MR 11,77a Zbl 0033.34803

[Schafer 1966] R. D. Schafer, An introduction to nonassociative algebras, Pure and Applied Mathematics 22, Academic Press, New York, 1966. MR 35 \#1643 Zbl 0145.25601 
[Tits 1955] J. Tits, "Sur certaines classes d'espaces homogènes de groupes de Lie", Acad. Roy. Belg. Cl. Sci. Mém. Coll. in $8^{\circ}$ 29:3 (1955), 268. MR 17,874f Zbl 0067.12301

[Tits 1962] J. Tits, "Une classe d'algèbres de Lie en relation avec les algèbres de Jordan", Nederl. Akad. Wetensch. Proc. Ser. A 65 (1962), 530-535. MR 26 \#3753 Zbl 0104.26002

[Truini and Biedenharn 1982] P. Truini and L. C. Biedenharn, "An $\mathscr{E}_{6} \otimes U(1)$ invariant quantum mechanics for a Jordan pair”, J. Math. Phys. 23:7 (1982), 1327-1345. MR 84j:81021 Zbl 0495.22012

[Truini et al. 1986] P. Truini, G. Olivieri, and L. C. Biedenharn, "Three graded exceptional algebras and symmetric spaces", Z. Phys. C 33:1 (1986), 47-65. MR 88c:17012 Zbl 0696.17005

[Zorn 1933] M. Zorn, "Alternativkörper und quadratische Systeme", Abh. Math. Semin. Hamb. Univ. 9 (1933), 395-402. Zbl 0007.05403

Received March 29, 2012.

Piero TRUini

DiPARTIMENTO DI FISICA

UNIVERSITÀ DI GENOVA

Via DodeCANeso 33

I-16146 GENOVA

ITALY

truini@ge.infn.it 


\title{
PACIFIC JOURNAL OF MATHEMATICS
}

\author{
http://pacificmath.org \\ Founded in 1951 by \\ E. F. Beckenbach (1906-1982) and F. Wolf (1904-1989)
}

\section{EDITORS}

V. S. Varadarajan (Managing Editor)

Department of Mathematics

University of California

Los Angeles, CA 90095-1555

pacific@math.ucla.edu

Vyjayanthi Chari

Department of Mathematics

University of California

Riverside, CA 92521-0135

chari@math.ucr.edu

\section{Robert Finn}

Department of Mathematics Stanford University

Stanford, CA 94305-2125

finn@math.stanford.edu

Kefeng Liu

Department of Mathematics

University of California

Los Angeles, CA 90095-1555

liu@math.ucla.edu
Darren Long

Department of Mathematics

University of California

Santa Barbara, CA 93106-3080

long@math.ucsb.edu

Jiang-Hua Lu

Department of Mathematics

The University of Hong Kong

Pokfulam Rd., Hong Kong jhlu@maths.hku.hk

Alexander Merkurjev

Department of Mathematics

University of California

Los Angeles, CA 90095-1555

merkurev@math.ucla.edu
Sorin Popa

Department of Mathematics University of California

Los Angeles, CA 90095-1555 popa@math.ucla.edu

Jie Qing

Department of Mathematics

University of California

Santa Cruz, CA 95064

qing@cats.ucsc.edu

Jonathan Rogawski

Department of Mathematics

University of California

Los Angeles, CA 90095-1555

jonr@math.ucla.edu

\section{PRODUCTION}

pacific@math.berkeley.edu

\section{SUPPORTING INSTITUTIONS}

ACADEMIA SINICA, TAIPEI

CALIFORNIA INST. OF TECHNOLOGY INST. DE MATEMÁTICA PURA E APLICADA KEIO UNIVERSITY

MATH. SCIENCES RESEARCH INSTITUTE NEW MEXICO STATE UNIV.

OREGON STATE UNIV.

\author{
STANFORD UNIVERSITY \\ UNIV. OF BRITISH COLUMBIA \\ UNIV. OF CALIFORNIA, BERKELEY \\ UNIV. OF CALIFORNIA, DAVIS \\ UNIV. OF CALIFORNIA, LOS ANGELES \\ UNIV. OF CALIFORNIA, RIVERSIDE \\ UNIV. OF CALIFORNIA, SAN DIEGO \\ UNIV. OF CALIF., SANTA BARBARA
}

\author{
UNIV. OF CALIF., SANTA CRUZ \\ UNIV. OF MONTANA \\ UNIV. OF OREGON \\ UNIV. OF SOUTHERN CALIFORNIA \\ UNIV. OF UTAH \\ UNIV. OF WASHINGTON \\ WASHINGTON STATE UNIVERSITY
}

These supporting institutions contribute to the cost of publication of this Journal, but they are not owners or publishers and have no responsibility for its contents or policies.

See inside back cover or pacificmath.org for submission instructions.

The subscription price for 2012 is US \$420/year for the electronic version, and \$485/year for print and electronic.

Subscriptions, requests for back issues from the last three years and changes of subscribers address should be sent to Pacific Journal of Mathematics, P.O. Box 4163, Berkeley, CA 94704-0163, U.S.A. Prior back issues are obtainable from Periodicals Service Company, 11 Main Street, Germantown, NY 12526-5635. The Pacific Journal of Mathematics is indexed by Mathematical Reviews, Zentralblatt MATH, PASCAL CNRS Index, Referativnyi Zhurnal, Current Mathematical Publications and the Science Citation Index.

The Pacific Journal of Mathematics (ISSN 0030-8730) at the University of California, c/o Department of Mathematics, 969 Evans Hall, Berkeley, CA 94720-3840, is published monthly except July and August. Periodical rate postage paid at Berkeley, CA 94704, and additional mailing offices. POSTMASTER: send address changes to Pacific Journal of Mathematics, P.O. Box 4163, Berkeley, CA 94704-0163.

PJM peer review and production are managed by EditFLOW ${ }^{\mathrm{TM}}$ from Mathematical Sciences Publishers.

PUBLISHED BY PACIFIC JOURNAL OF MATHEMATICS

at the University of California, Berkeley 94720-3840

A NON-PROFIT CORPORATION

Typeset in LATEX

Copyright $(02012$ by Pacific Journal of Mathematics 


\section{PACIFIC JOURNAL OF MATHEMATICS}

Volume $260 \quad$ No. $1 \quad$ November 2012

The decomposition of global conformal invariants: Some technical proofs II

SPYROS ALEXAKIS

On deformation quantizations of hypertoric varieties

GWYN BELLAMY and TOSHIRO KUWABARA

Almost factoriality of integral domains and Krull-like domains

GYU Whan CHANG, HWANKOO KIM and JUNG WOOK LIM

Singularities of free group character varieties

CARLOS FLORENTINO and SEAN LAWTON

Energy identity for the maps from a surface with tension field bounded 181 in $L^{p}$

LI JIAYU and ZHU XIANGRONG

Remarks on some isoperimetric properties of the $k-1$ flow

YU-CHU LIN and DONG-Ho TSAI

Demystifying a divisibility property of the Kostant partition function 215

KAROLA MÉSZÁros

Exceptional Lie algebras, SU(3), and Jordan pairs

PIERO TRUINI

Lower estimate of Milnor number and characterization of isolated homogeneous hypersurface singularities

Stephen S.-T. Yau and HuAiqing Zuo 